Received: 15 November 2016

Accepted: 6 March 2017

Published online: 30 March 2017

\section{OPEN Muscle-specific downregulation of GR levels inhibits adipogenesis in porcine intramuscular adipocyte tissue}

Weiwei Chu ${ }^{1,2}$, Wei Wei ${ }^{1}$, Haiyin $\mathrm{Han}^{1}$, Ying Gao ${ }^{3}$, Kaiqing Liu ${ }^{1}$, Ye Tian $^{1}$, Zaohang Jiang ${ }^{1}$, Lifan Zhang $^{1}$ \& Jie Chen ${ }^{1}$

Intramuscular adipose is conducive to good pork quality, whereas subcutaneous adipose is considered as waste in pig production. So uncovering the regulation differences between these two adiposes is helpful to tissue-specific control of fat deposition. In this study, we found the sensitivity to glucocorticoids (GCs) was lower in intramuscular adipocytes (IMA) compared with subcutaneous adipocytes (SA). Comparison of glucocorticoid receptor (GR) revealed that IMA had lower GR level which contributed to its reduced GCs sensitivity. Higher methylation levels of GR promotor 1-C and 1-H were detected in IMA compared with SA. GR expression decrease was also found in adipocytes when treated with muscle conditioned medium (MCM) in vitro, which resulted in significant inhibition of adipocytes proliferation and differentiation. Since abundant myostatin (MSTN) was detected in MCM by ELISA assay, we further investigated the effect of this myokine on adipocytes. MSTN treatment suppressed adipocytes $G R$ expression, cell proliferation and differentiation, which mimicked the effects of MCM. The methylation levels of GR promotor 1-C and 1-H were also elevated after MSTN treatment. Our study reveals the role of $G R$ in muscle fiber inhibition on intramuscular adipocytes, and identifies myostatin as a muscle-derived modulator for adipose GR level.

Adipose tissue is a large endocrine organ in the body, and functions in energy storage, hormone production, and immune function ${ }^{1}$. Most adipose tissues are deemed as waste in meat animal production, hence diminishing body fat content has long been an important goal of pig breeding. Consequently, subcutaneous adipose tissue (SAT), the major part of body fat, is supposed to be in the smallest amount possible in pig production. However, intramuscular adipose tissue (IMAT) is exceptionally regarded as favorite fat because it is conducive to pork quality. Moderate intramuscular fat content can promote pork juiciness, tenderness and flavor. Therefore, the breeding directions of IMAT and SAT are opposite, being positive for IMAT while negative for SAT. Unfortunately, reducing SAT always results in parallel decline of IMAT, which occurs prevalently in modern lean pig breeds. The reason might be that the regulation mechanisms for various fat tissues should be of similarities. Even they are from different fat depots. This explains why IMAT and SAT are of moderate positive correlation ${ }^{2}$.

However, adipose tissues from different locations also display their special physiological and biochemical characteristics. Many studies reported that subcutaneous adipocytes (SA) grew faster ${ }^{3}$ and accumulated more lipids $^{4}$ than intramuscular adipocytes (IMA). Besides, the manners of their substrate utilization are different. The IMA consume more glucose, while SA mainly utilize exogenic fatty acids ${ }^{5}$. What's more, differences of cell sizes ${ }^{6}$, secretory functions $s^{7,8}$, levels of genes expression ${ }^{9}$, and hormone sensitivities ${ }^{10,11}$ were observed between SA and IMA. Since the requirements for these two fat tissues are different, understanding the molecular regulation difference between IMAT and SAT is of great significance to pig breeding.

Fat deposition is a complex process involving proliferation and differentiation of adipocytes. Glucocorticoids (GCs) play vital roles in promoting fat deposition via inducing proliferation ${ }^{12,13}$ and differentiation ${ }^{14,15}$ of

${ }^{1}$ College of Animal Science and Technology, Nanjing Agricultural University, Nanjing, 210095, P.R. China. ${ }^{2}$ Precision Medicine and Healthcare, Tsinghua-Berkeley Shenzhen Institute, Shenzhen, 518055, P.R. China. ${ }^{3}$ College of Veterinary Medicine, Nanjing Agricultural University, Nanjing, 210095, P.R. China. Weiwei Chu and Wei Wei contributed equally to this work as co-first authors. Correspondence and requests for materials should be addressed to J.C. (email: jiechen@njau.edu.cn) 

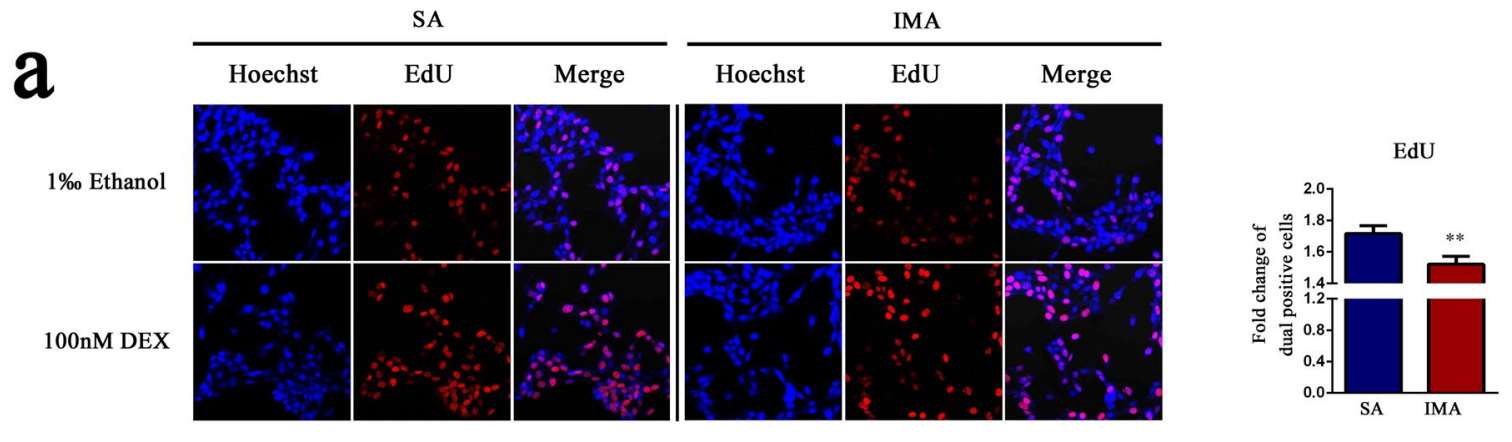

b
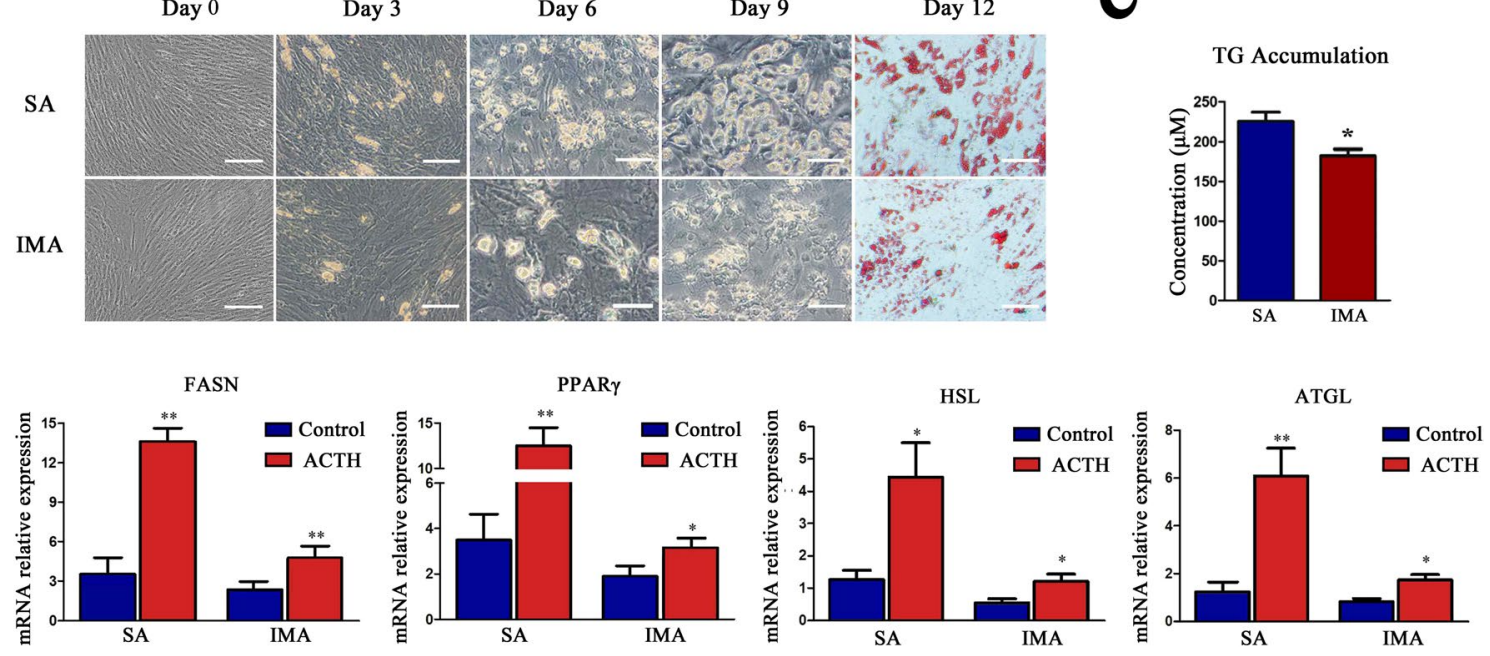

Figure 1. IMA was less sensitive to GCs than SA. (a) The EdU assay of SA and IMA pre-adipocytes. The porcine SA and IMA pre-adipocytes were treated with $1 \%$ ethanol or $100 \mathrm{nM}$ DEX for 3 days, then the proliferating nuclei were stained red with EdU, the nuclei of all cells were stained blue with Hoechst for 2 hours. Three random pictures per group from confocal microscopy were used to count the cell numbers of EdU positive cells and Hoechst positive cells, and the ratio of the EdU positive cells to Hoechst positive cells was calculated in each picture. Data are shown as the mean \pm SEM, $\mathrm{n}=3$ per group, $* P<0.05$. (b) The differentiation of SA and IMA pre-adipocytes after induced with DEX. Cells were induced to differentiation by $2.5 \mu \mathrm{M}$ DEX, and imaged by inverted microscope. Scale bar $=100 \mu \mathrm{m}$. (c) Triglyceride accumulation in SA and IMA treated as (b) was detected on day 9. Data are shown as the mean \pm SEM, $\mathrm{n}=3$ per group, $* P<0.05$. (d) mRNA expression of lipid metabolism genes in SA and IMA tissues of sows injected with $1 \mathrm{U} / \mathrm{kg}$ ACTH $(\mathrm{n}=6)$ or equivalent volume saline $(n=6)$ intravenously per day for a total 9 days were detected. *Means significant differences between control and ACTH groups, $* P<0.05$, $* * P<0.01$.

pre-adipocytes. And the adipocytes GCs sensitivity decides the ability of fat deposition. In human, visceral adipocytes (VA) are more sensitive to GCs than $\mathrm{SA}^{16}$. When the levels of GCs are induced, more lipids will deposit in VA, leading to "central obesity"16-20. However, the molecular mechanism underlying differential GCs sensitivities of various adipose tissues still remains unknown.

Basing on these findings, we hypothesize that the physiological differences between SA and IMA might be caused by the muscle specific regulation of adiposes. In current study, we investigate to learn whether GCs sensitivity is involved in the physiological differences between SA and IMA, and whether the local muscle-to-fat regulation of GRs is responsible for muscle-specific GCs sensitivity. Our results demonstrated a novel pathway for the adverse effects of skeletal muscle on adipocytes, which could contribute to specifically regulate IMA in pork quality improvement.

\section{Results}

IMA was less sensitive to GCs than SA. Many studies demonstrated that GCs played important roles in adipocytes proliferation ${ }^{14,15}$ and differentiation ${ }^{12,13}$. Adipose tissue with higher GCs sensitivity tends to deposit more lipids ${ }^{10,20}$. However, the GCs sensitivity of IMA is still unknown. We compared GCs sensitivity between porcine IMA and SA in vitro and in vivo. SA and IMA pre-adipocytes were treated with 10, 100 and $1000 \mathrm{nM}$ dexameson (DEX, GCs mimics) respectively. Cell viability assessment results showed that all three concentrations of DEX could promote the proliferation of SA and IMA pre-adipocytes, of which $100 \mathrm{nM}$ DEX treatment is of the highest efficiency. When treated with $100 \mathrm{nM}$ DEX for 24, 48 and 72 hours, IMA exhibited significantly lower viability than SA pre-adipocytes $(P<0.05)$ (Supplementary Fig. 1). After $100 \mathrm{nM}$ DEX treated for 48 hours, EdU assay results indicated that the dual positive cells (proliferating cells) were significantly less in IMA than in SA pre-adipocytes $(P<0.01)$ (Fig. 1a). 
a

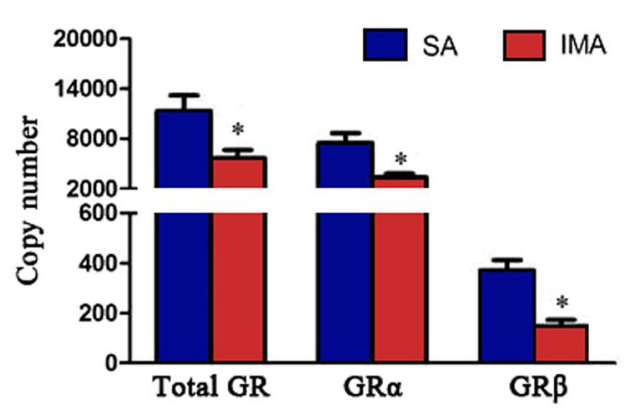

C

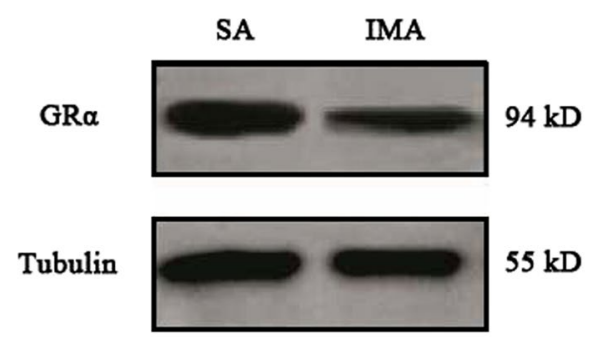

b
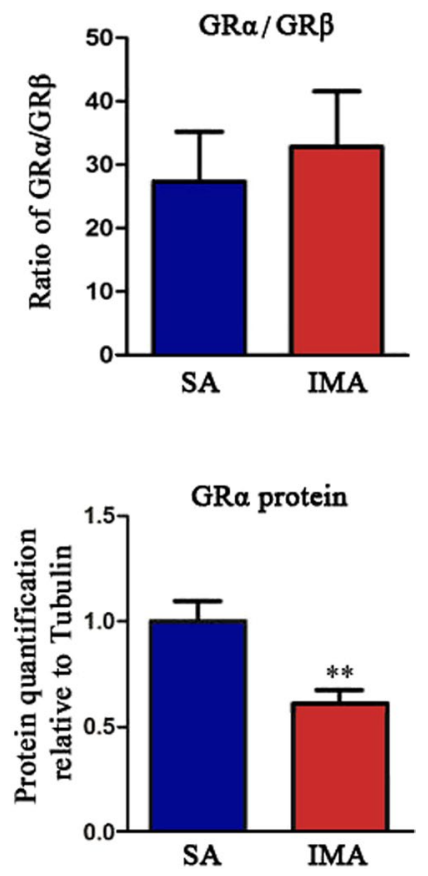

Figure 2. GR levels in IMA were lower than SA. Backfat and longissimus dorsi muscles were from 12 Erhualian sows, then the adipose tissues were collected for RNA and protein isolation. (a) Copy numbers of total GR, GR $\alpha, G R \beta$ mRNA in SA and IMA tissues were detected by RT-qPCR. (b) The ratio of GR $\alpha / G R \beta$ was displayed according to the result obtained in (a). Data are shown as the mean \pm SEM, $\mathrm{n}=12$ per group, $* P<0.05$. (c) Expression of GR $\alpha$ protein in SA and IMA tissues was detected by Western blot (left), and the relative protein expression level was displayed as column charts (right). $\mathrm{n}=3$ per group, $* * P<0.01$.

DEX effect on the differentiation of SA and IMA pre-adipocytes was compared by treating pre-adipocytes with $2.5 \mu \mathrm{M}$ DEX, which had been proved to be the optimal concentration for pre-adipocytes differentiation inducement $^{21}$. On day 0 of treatment, SA and IMA pre-adipocytes were fibrous cells. During the process of differentiation, more and more lipid droplets were formed in both fat cells, but smaller and less lipid droplets were observed in IMA cells according to Oil Red O staining result (Fig. 1b). Results of triglyceride (TG) accumulation detection showed that TG concentration was also lower in IMA than in SA $(P<0.05)$ (Fig. $1 \mathrm{c})$.

Adrenocorticotropic hormone (ACTH) stimulates the adrenal cortex to synthesize and secrete GCs. In this study, ACTH was injected intravenously for 9 days to compare GCs sensitivity of SA and IMA tissues in vivo. The result showed that administration of ACTH increased average plasma cortisol level by 2.2 folds (Supplementary Fig. 2). Fatty acid synthase (FASN) and peroxisome proliferator activated receptor $\gamma$ (PPAR $\gamma$ ) have key effects on the adipogenesis ${ }^{22,23}$, and hormone sensitive lipase (HSL) and adipose triglyceride lipase (ATGL) are the maker genes of adipolysis ${ }^{24,25}$. These genes were the downstream genes of the GR pathway, directly representing the status of the lipid metabolism. Here the elevated endogenous GCs significantly increased the expressions of these lipid metabolism genes in both SA and IMA tissues $(P<0.05)$ (Fig. 1d). More importantly, the mRNA expression fold changes of FASN [3.9(SA) vs. 2.1(IMA)], PPAR $\gamma$ [5.2(SA) vs. 2.2(IMA)], HSL [3.5(SA) vs. 2.0(IMA)], ATGL [5.2(SA) vs. 1.9(IMA)] were significantly less in IMA than in SA tissues (Supplementary Fig. 3).

GR $\alpha$ was expressed less in IMA than SA. Circulating GCs can't work as physiological factors without its receptor GR. GR $\alpha$ is the primary functional isoform of GR, while GR $\beta$ is the inhibitor of GR $\alpha$. In order to reveal the mechanism of the different GCs sensitivities, we detected the GR expression levels of SA and IMA in vivo. SA and IMA tissues were obtained from 12 Erhualian pigs, and RT-qPCR results showed that total GR, GR $\alpha$ and GR $\beta$ mRNA were expressed less in IMA than in SA tissues (Fig. 2a), but the ratio of GR $\alpha / G R \beta$ mRNA was no significant difference (Fig. 2b). The western blot analyses revealed that GR $\alpha$ protein was less in IMA than in SA tissues (Fig. 2c). These data indicated that GR $\alpha$ was the key factor for the differences of GCs sensitivities between SA and IMA.

GR $\alpha$ levels were mainly determined by variants E1-C and E1-H. The gene structure of GR is very complex, because the no-coding exon 1 contains different kinds of alternative splicing variants. To further identify which exon 1 variants mainly contribute to the different expression levels of GR $\alpha$ between SA and IMA, a total of nine types of exon 1 variants were detected in SA and IMA tissues. The results showed that E1-C was the dominated type, accounting for $65.3 \sim 76.4 \%$ of the total GR mRNA. Thus, E1-C had a maximum effect on the total GR expression. Expression levels of E1-F, E1-D, E1-B, E1-A and E1-H were medium (1.6 10.2\%), and 
a

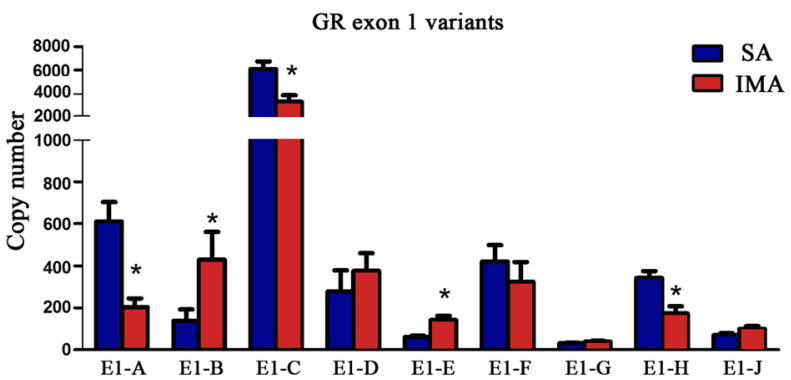

b

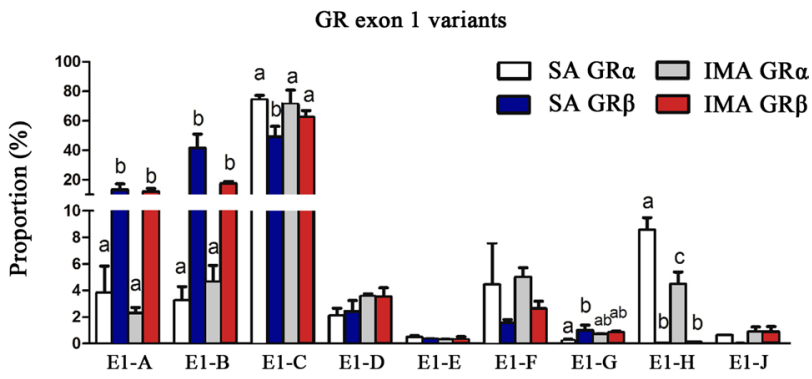

Figure 3. Expression patterns of GR exon 1 variants. (a) Copy number of GR exon 1 variants in SA and IMA tissues. $\mathrm{n}=12$ per group, $* P<0.05$. (b) Variants proportion of $\mathrm{GR} \alpha$ and GR $\beta$ in SA and IMA tissues. $\mathrm{n}=6$ per group, within column the different lowercases mean significant difference (One-way ANOVO, $P<0.05$ ) within the columns, and the same lowercase means no significant difference (One-way ANOVO, $P>0.05$ ).

the expression level of E1-E, E1-J and E1-G were slight (0.4 2.8\%). Notably, E1-A, E1-C and E1-H were less expressed in IMA than SA tissues (Fig. 3a). For GR $\alpha$ and GR $\beta$, the expression patterns of nine variants were not the same. Expressions of E1-A and E1-B were significantly higher in GR $\beta$ than in GR $\alpha$, indicating that E1-A and E1-B tend to have more effects on the GR $\beta$ expression. Whereas E1-H was detected only in GR $\alpha$, suggesting that E1-H transcript only encodes GR $\alpha$ in SA and IMA tissues. Moreover, E1-H level in SA was 1.9 times as high as that in IMA tissues (Fig. 3b). Therefore, higher levels of GR $\alpha$ in SA were mainly due to the higher levels of E1-C (the dominated variant) and $\mathrm{E} 1-\mathrm{H}$ (GR $\alpha$ specific variant).

Muscle conditioned medium suppressed adipocytes proliferation and differentiation by reducing GR $\alpha$ expression. From the above results we concluded that the less expression of GR $\alpha$ variants E1-C and E1-H in IMA mainly cause its lower sensitivity of GCs compared with SA. Considering its special location, we assumed that the GR $\alpha$ levels in IMA were influenced by muscles. In order to mimic the effects of muscle fibers on normal adipocytes (haven't been influenced by muscle), we used skeletal muscle conditioned medium (MCM) to treat subcutaneous pre-adipocytes. The results showed that the levels of variants E1-C and E1-H were reduced by $32.6 \%$ and $73.3 \%$ respectively, and GR $\alpha$ level was reduced by $46.4 \%$ on day 3 of treatment (Fig. 4 a,b). Cell counting revealed that the cells number in MCM group was decreased by $41 \%$ compared with the control group. Treating pre-adipocytes with $100 \mathrm{nM}$ RU486, a GR antagonist, had similar inhibitory effects, as the number of cells decreased by $38 \%$. Notably, $100 \mathrm{nM}$ DEX could rescue the inhibitory effects of MCM on the pre-adipocytes proliferation, as the cells number in MCM + DEX group was significantly larger than MCM group $(P<0.05)$ (Fig. 4c). On day 12 of inducing differentiation, Oil red O staining displayed that MCM inhibited the differentiation of pre-adipocytes. TG assay showed that the TG concentration decreased by $45 \%$ (Fig. 4d). Moreover, the late apoptosis rate of pre-adipocytes increased 3.3-fold, and cell necrosis rate increased 8.0-fold, which indicated that MCM promoted the apoptosis of pre-adipocytes. RU486 had a similar effect on pre-adipocytes apoptosis, which increased late apoptosis rate by 2.4 -fold, and cell necrosis rate by 6.8 -fold. Adding DEX to MCM could rescue the effects of MCM on the pre-adipocytes apoptosis (Fig. 4e).

Myostatin inhibited adipogenesis and reduced $\mathrm{GR} \alpha$ expression in adipocytes. Myostatin (MSTN) is a special myokine secreted by muscle. High concentration of MSTN was also detected in MCM in this study (Supplementary Fig. 4). We previously found that MSTN could inhibit the differentiation of pre-adipocytes $^{26}$. Therefore, we presumed that MSTN contributes to the MCM inhibiting effect on intramuscular adipocytes. Firstly, we assessed the effect of MSTN on adipogenesis. After treating subcutaneous pre-adipocytes with $50 \mathrm{ng} / \mathrm{ml}$ MSTN for 2 days, we found the proportion of EdU positive cells decreased by $31 \%$ (Fig. $5 \mathrm{a}$ ), indicating that MSTN could suppress pre-adipocytes proliferation. To detect the effects of MSTN on differentiation of pre-adipocytes, the pre-adipocytes were cultured in culture medium until full confluence, and then the cells were induced differentiation with or without $50 \mathrm{ng} / \mathrm{ml}$ MSTN for 9 days. Oil red O staining and TG assay revealed that MSTN significantly inhibited pre-adipocytes differentiation, as the TG concentration in adipocytes decreased $46 \%$ after MSTN treatment (Fig. 5b). 
$\mathbf{a}$

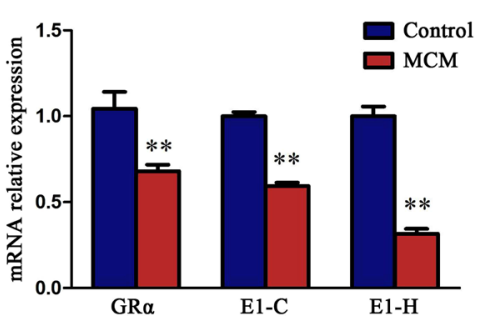

c

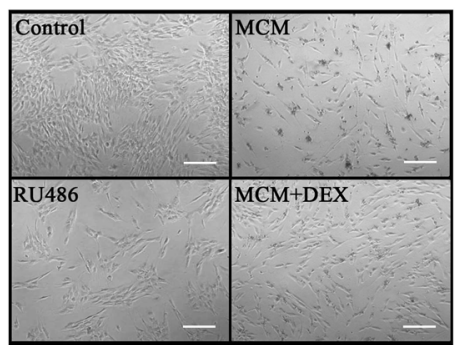

d

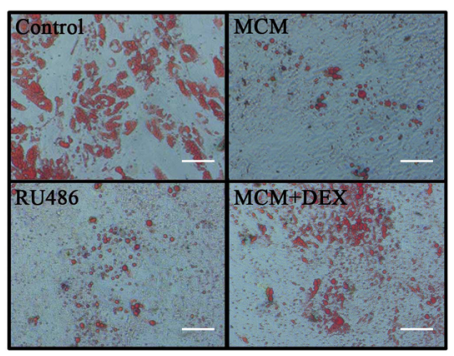

e

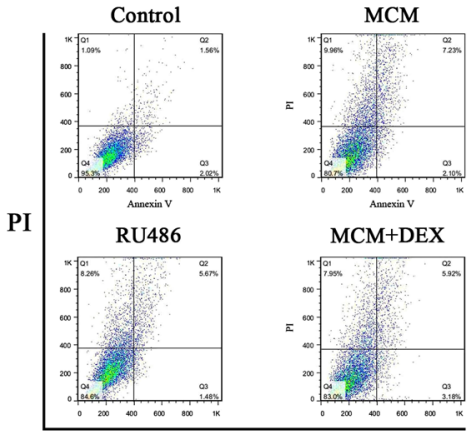

Annexin V
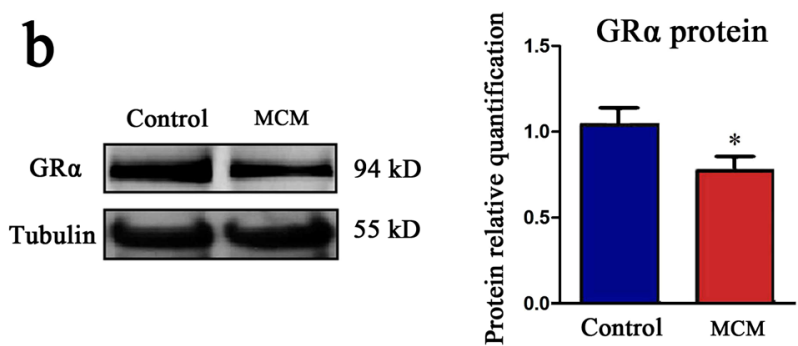

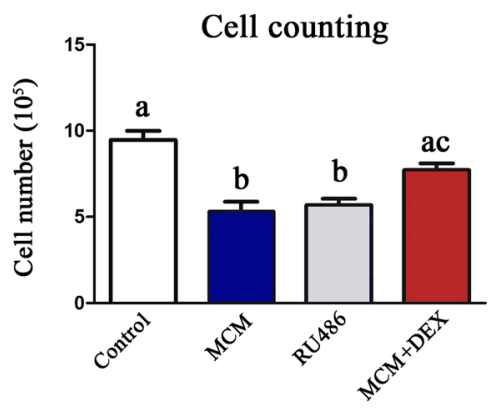

TG accumultion

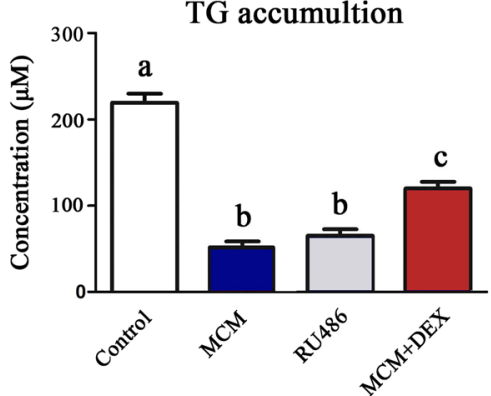

Apoptosis rate

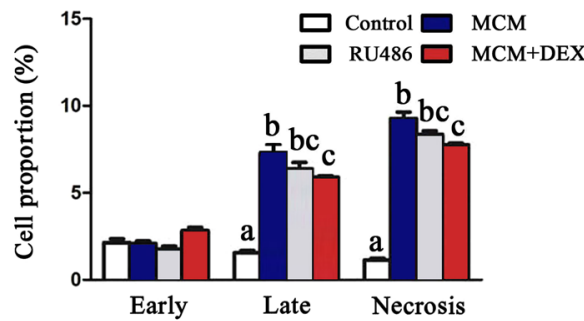

Figure 4. Skeletal muscle conditioned medium inhibited the adipogenesis by reducing GR $\alpha$ expression. The subcutaneous pre-adipocytes were cultured with skeletal muscle conditioned medium (MCM) or ordinary medium for 3 days, then the cells were collected for RNA, protein isolation and flow cytometry assay. (a) The mRNA expression of GR $\alpha$ and E1-C, E1-H was detected by RT-qPCR, $n=6$ per group. (b) Expression of GR $\alpha$ protein was detected by Western blot (left) and displayed as column charts after quantification (right), $\mathrm{n}=3$ per group, ${ }^{*} P<0.05$. (c) The effects of MCM on the proliferation of pre-adipocytes were detected by cell imaging (left) and cell counting (right). Cells were cultured with normal culture medium, MCM medium, normal culture medium with $100 \mathrm{nM}$ RU486, or $100 \mathrm{nM} \mathrm{DEX}+\mathrm{MCM}$ medium respectively, $\mathrm{n}=6$ per group. The different lowercases mean significant difference (One-way ANOVO, $P<0.05$ ) within the columns, and the same lowercase means no significant difference (One-way ANOVO, $P>0.05$ ), the same below. (d) The effects of MCM on the pre-adipocytes differentiation. After reaching confluence, pre-adipocytes were induced to differentiation for 12 days, then the Oil-red O staining (left) and TG assay (right) were conducted. $n=3$ per group. (e) The effects of MCM on the apoptosis of pre-adipocytes were detected by flow cytometry (left) and displayed as column charts after quantification (right), $\mathrm{n}=3$ per group. 
a

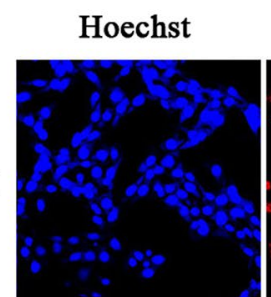

EdU

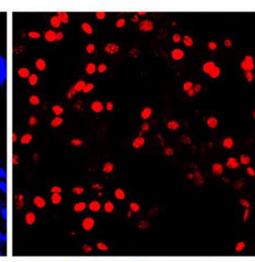

MSTN

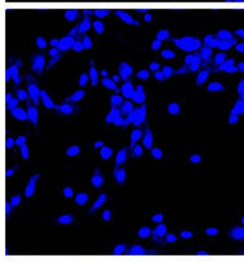

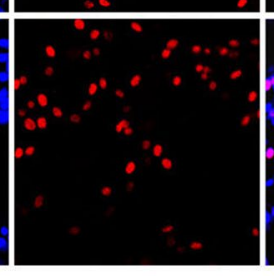

b
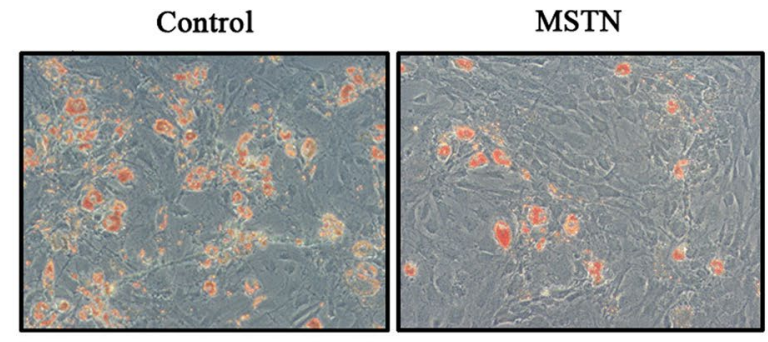

\section{C}

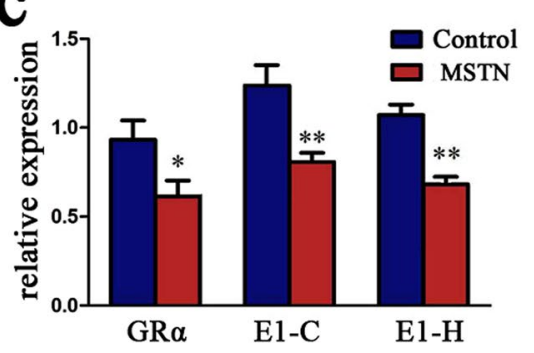

Merge
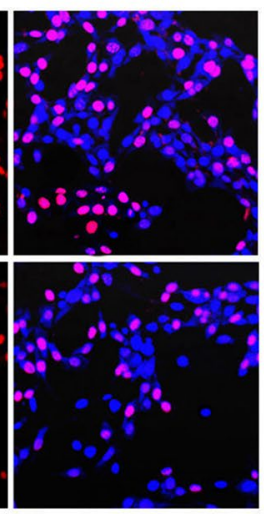

MSTN

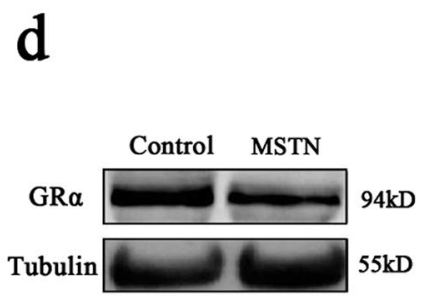

Figure 5. MSTN inhibited adipogenesis and reduced GR $\alpha$ expression. (a) The effects of MSTN on the proliferation of subcutaneous pre-adipocytes. Pre-adipocytes were treated with $50 \mathrm{ng} / \mathrm{ml} \mathrm{MSTN}$ for 2 days, then the proliferating nuclei were stained red with EdU for 2 hours, and nuclei of all cells were stained blue with Hoechst (left). Three random pictures per group from confocal microscopy were used to count the cell numbers of EdU positive cells and Hoechst positive cells, and the ratio of the EdU positive cells to Hoechst positive cells was calculated in each picture (right). (b) Oil red O staining (left) and TG assay (right), $\mathrm{n}=3$ per group. The pre-adipocytes were cultured in culture medium until full confluence, then the cells were induced to differentiation with PBS or $50 \mathrm{ng} / \mathrm{ml}$ MSTN for 9 days. (c) The mRNA expression of GR $\alpha$, exon $1 \mathrm{C}$ and $1 \mathrm{H}$ was detected by RT-qPCR, $\mathrm{n}=6$ per group. (d) The protein expression of GR $\alpha$ was detected by Western blot (left) and displayed as column charts after quantification (right), $\mathrm{n}=3$ per group. $* P<0.05 ; * P<0.01$.

We further aim to detect whether MSTN play a role in the muscle fiber inhibition on IMA GR expression. After treated with $50 \mathrm{ng} / \mathrm{ml}$ MSTN for 3 days, the mRNA levels of GR $\alpha$, E1-C and E1-H in subcutaneous pre-adipocytes were reduced by $35.8 \%, 34.5 \%$ and $33.4 \%$ respectively (Fig. $5 \mathrm{c}$ ), and the levels of GR $\alpha$ protein decreased by $24 \%$ compared with the control group (Fig. $5 \mathrm{~d}$ ).

Myostatin influenced the cell cycles and apoptosis of adipocytes. After treated with MSTN for 2 days, Flow cytometry was used to detect the cell cycles and apoptosis of pre-adipocytes. The results showed that proportion of subcutaneous pre-adipocytes in G0-G1 phase was increased by $8.3 \%$, and cells in S phase decreased by $5.1 \%$, suggesting that MSTN arrested pre-adipocytes in the G0-G1 phase (Fig. 6a). Besides, early and late apoptosis rates were increased by $220 \%$ and $90 \%$ respectively (Fig. 6b), indicating the promotion role of MSTN for pre-adipocytes apoptosis.

Higher methylation levels of GR promoter $1-\mathrm{C}$ and 1- $\mathrm{H}$ were detected in IMA. DNA methylation modification is one of important ways to regulate GR expression ${ }^{27,28}$, and thus we analyzed the methylation levels of GR promoter in SA and IMA tissues. The locations of variant E1-C and E1-H promoters were showed in 
a

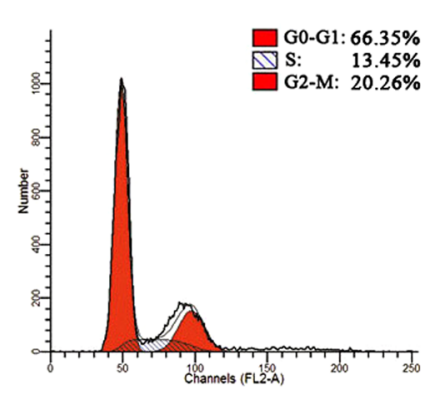

b

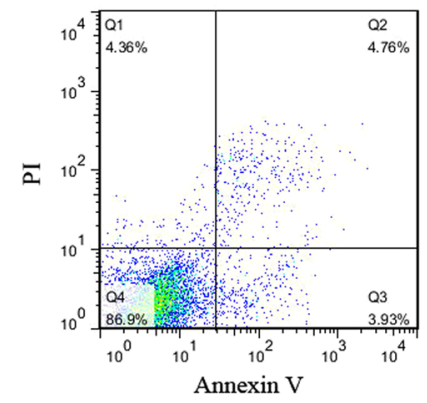

MSTN

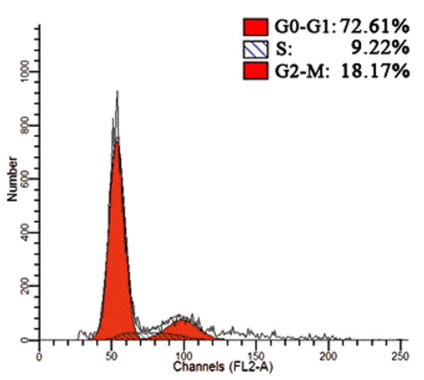

MSTN

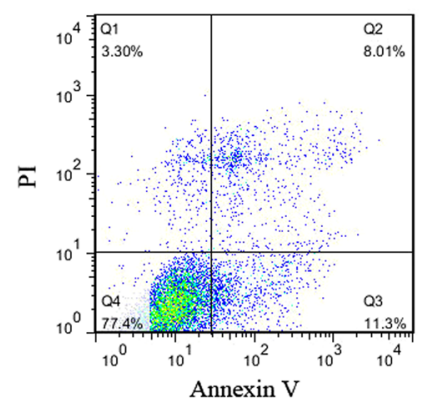

Cell cycle

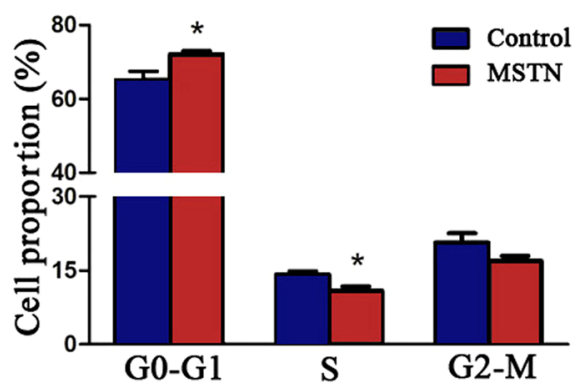

Apoptosis rate

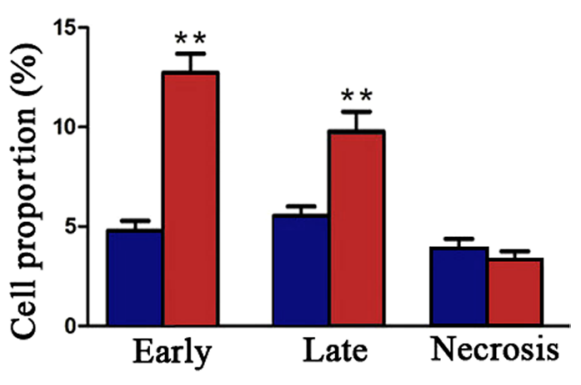

Figure 6. MSTN influenced cell cycles and apoptosis of adipocytes. Subcutaneous pre-adipocytes were treated with $50 \mathrm{ng} / \mathrm{ml}$ MSTN for 2 days and then harvested for further detection. (a) The effects of MSTN on the cell cycles of pre-adipocytes. Cells were stained with PI, then the cell cycles were detected by flow cytometry (left) and subsequently displayed as column charts after quantification (right), $\mathrm{n}=3$ per group. (b) The effects of MSTN on the apoptosis of pre-adipocytes. Cells were stained with PI and Annexin V, then the apoptosis rate was detected by flow cytometry (left) and displayed as column charts after quantification (right). $\mathrm{n}=3$ per group, $* P<0.05 ; * * P<0.01$.

Fig. $7 \mathrm{a}-\mathrm{c}$, and the $\mathrm{CpG}$ sites were named CpG1 55 according to their orders in sequence. Bisulfite sequencing results showed that the methylation level of promoter 1-C was $46.7 \%$ in IMA pre-adipocytes, which was significantly higher than that in SA pre-adipocytes $(32.6 \%)(P<0.05)$ (Fig. $7 \mathrm{~d})$. For promoter $1-\mathrm{H}$, the methylation level in IMA $(15.1 \%)$ was significantly higher than that in SA pre-adipocytes $(9.2 \%)(P<0.05)$ (Fig. 7e).

Myostatin elevated the methylation levels of promoter $1-\mathrm{C}$ and $1-\mathrm{H}$. We further detected whether MSTN influence methylation levels of GR promoter in pre-adipocytes by DNA methylation immunization (MeDIP). After treated by $50 \mathrm{ng} / \mathrm{ml}$ MSTN for 2 days, the methylation levels of promoter $1-\mathrm{C}$ increased from $15 \%$ to $29 \%$, and the methylation levels of promoter $1-\mathrm{H}$ increased from $15 \%$ to $26 \%$ (Fig. $8 \mathrm{a}$ ). RT-qPCR results demonstrated that DNMT3 $\alpha$ was upregulated when treated by MSTN (Fig. 8b).

\section{Discussion}

Distribution of body fat is an important predictor of metabolic abnormalities, because subcutaneous adipose is inversely associated with glucose and lipid concentrations, while increased intramuscular adipose is independently associated with insulin resistance and $\mathrm{T} 2 \mathrm{D}^{29,30}$. Previous studies indicated that the proliferation and differentiation abilities, levels of genes involved in lipid metabolism were dramatically lower in IMA than SA $\mathrm{A}^{7,9,31,32}$. Unclosing the mechanism of the lower ability of adipogenesis in IMA is of great medical and economic value. As we know, glucocorticoids (GCs) is a key factor to regulate the proliferation ${ }^{12,13}$ and differentiation ${ }^{14,15}$ of adipocytes. Adipose with higher GCs sensitivity tend to deposit more lipids ${ }^{10,20}$. For example, the visceral adipose is more sensitive to GCs than subcutaneous adipose ${ }^{16}$, and when the levels of GCs were elevated, more lipids would deposit in visceral adipocytes, leading to "central obesity"17-19. In present study, we tend to uncover why the lipids prone to deposit less in IMA, which could be helpful to regulating IMA specially in pork quality improvement. Our results indicated that the GCs induced-proliferation, differentiation and the marker genes' expression were lower in IMA compared with SA, that lead to less lipids were deposited in IMA.

Physiological effects of circulating GCs are mediated by the GR. So it is reasonable that the levels of GR influence GCs sensitivity, and it is confirmed by lots of studies. Cortisol resistance is accompanied by a reduction of $\mathrm{GR}^{33,34}$, however high levels of GR increased GCs sensitivity ${ }^{35}$. GR $\alpha$ is a hormone-activated transcription factor, while GR $\beta$ is transcriptionally inactive and is a potential inhibitor of activated GR $\alpha^{36}$. The ratio of GR $\alpha / G R \beta$ usually influences the GCs sensitivity. In present study, lower levels of GR mRNA and protein were detected in IMA, but the ratio of GR $\alpha / G R \beta$ was no difference between IMA and SA. Thus, so we only took GR $\alpha$ as our target in next study. 
a

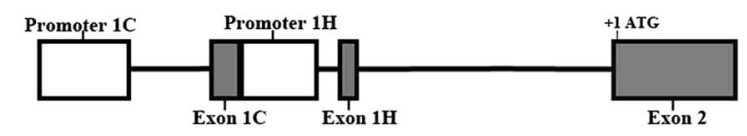

b
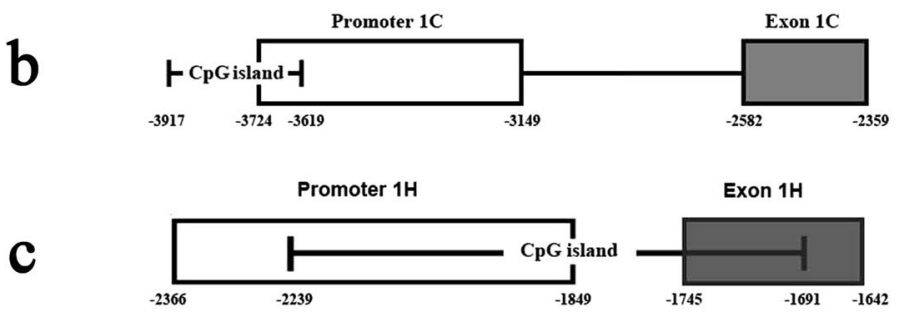

d

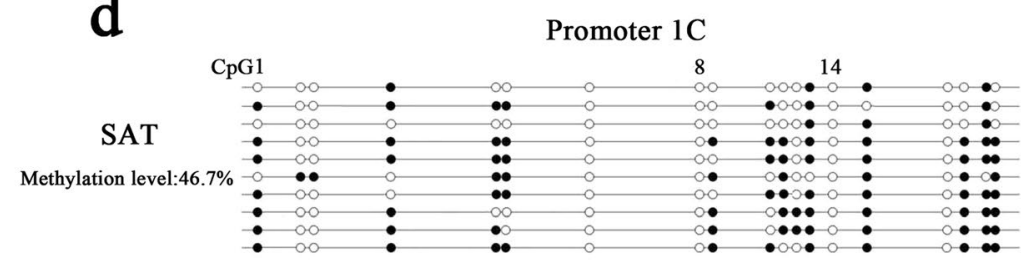

IMAT

Methylation level: $32.6 \%$

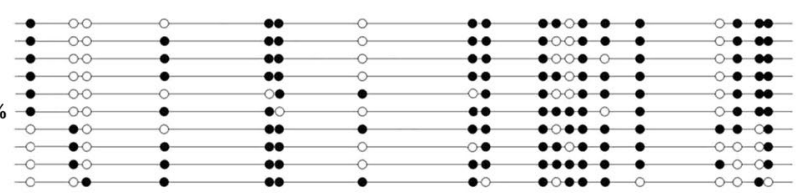

e

Promoter $1 \mathrm{H}$

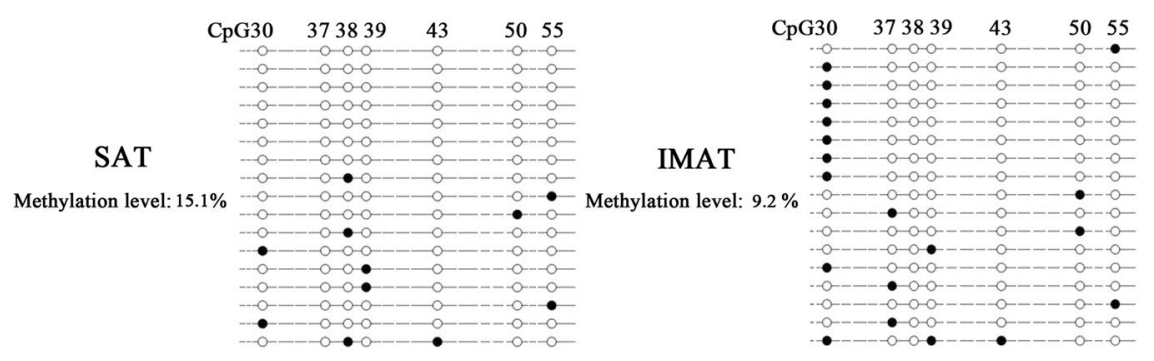

Figure 7. Methylation levels of promoter 1-C and 1-H were higher in IMA. (a) Locations of promoter 1-C and 1-H. The gray vertical bars represent the exons of GR gene; the open bar represents the promoter. (b) Location of $\mathrm{CpG}$ island in promoter 1-C. (c) Location of CpG islands in promoter 1-H. Nucleotide numbering is relative to +1 at the initiating ATG codon. (d) Methylation status of promoter 1-C in IMA and SA. (e) Methylation status of promoter 1-H in IMA and SA. Each line represents an individual bacterial clone that was sequenced. Open circles indicate unmethylated CpG sites. Black circles indicate methylated CpG sites.

What's more, the isoforms of the GR were influenced by the expression of alternative exon 1 variants in different species and tissues ${ }^{37-40}$. To uncover the primary exon 1 variants in dominating the tissue specific expression of GR $\alpha$, we measured the express patterns of nine GR variants in SA and IMA. The results showed that E1-C was the highest expressed variants, and expressions of E1-A, E1-C and E1-H were significantly lower in IMA. Further study on the express patterns of variants for GR $\alpha$ and GR $\beta$ showed that E1-A mainly existed in GR $\beta$ other than GR $\alpha$. Notably, E1-H was only detected in GR $\alpha$, which means the E1-H does not contribute to GR $\beta$ expression. Therefore, the lower level of GR $\alpha$ in IMA was mainly caused by the lower expression of E1-C (the primary variant) and E1-H (GR $\alpha$ specific variant). Previous reports disclosed that E1-C account for a proportion of $70 \%$ to $80 \%$ in different porcine tissues ${ }^{40,41}$, and the mini genes which contain $\mathrm{E} 1-\mathrm{H}$ increased the proportion of $\mathrm{GR}^{42}$, which is coincident with our present results.

Most studies of muscle-adipose crosstalk were focused on the effects of adipose on the muscle, only a few studies reported that myocytes suppressed differentiation of adipocytes ${ }^{43}$, 44 , and degenerating myofibres could affected the cellular morphology of 3T3-L1 preadipocytes ${ }^{45}$. However, the mechanism was still unknown. Considering the special location of IMA, we assumed GR $\alpha$ level in IMA influenced by muscles. We treated porcine adipocytes with MCM to mimic the effects of muscle on the adipocytes in vitro, finding that the expression of E1-C, E1-H and GR $\alpha$ was inhibited, which confirmed our hypothesis. Along with the reduction of GR $\alpha$, the proliferation and differentiation of adipocytes were also suppressed, and the apoptosis was induced. The similar effects were observed when adipocytes were treated with RU-486 (GR antagonist), and DEX could partly recover 
a

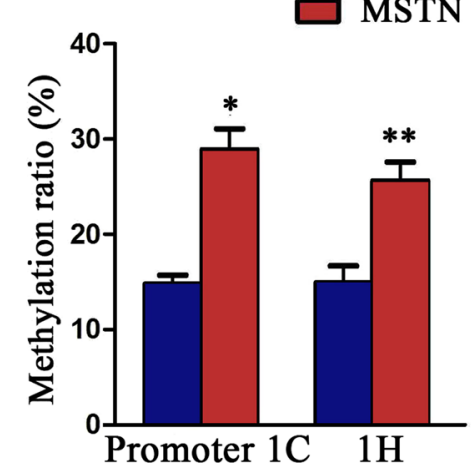

b
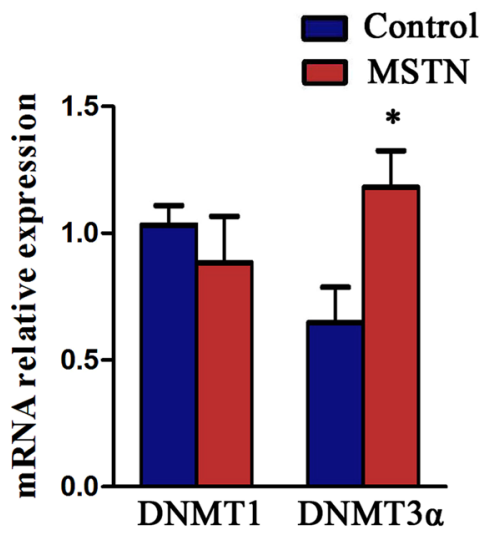

Figure 8. MSTN elevated the methylation levels of promoter $1-\mathrm{C}$ and $1-\mathrm{H}$. After treated with $50 \mathrm{ng} / \mathrm{ml}$ MSTN for 2 days, the pre-adipocytes were harvested, then the methylation status of promoter 1-C and $1-\mathrm{H}$ were detected by methylated DNA immunoprecipitation method (a), and the relative mRNA expression of DNA methyltransferase DNMT1 and DNMT3 $\alpha$ were detected by RT-qPCR $(\mathbf{b}) . \mathrm{n}=6$ per group. $* P<0.05$; $* * P<0.01$.

the negative effects of MCM. These data coincides with the previous studies, and unclosed that MCM inhibited adipogenesis by reducing GR.

In our previous study, we co-cultured 3T3-L1 pre-adipocytes with $\mathrm{C} 2 \mathrm{C} 12$ myotubes by trans-well inserts, and found that $\mathrm{C} 2 \mathrm{C} 12$ cells inhibited the proliferation and differentiation of 3T3-L1 cells ${ }^{46}$. Muscle has been identified as an endocrine organ owing to its capacity to produce and secrete a variety of myokines and other proteins ${ }^{47}$. Some cytokines including IL-1 $\alpha$, IL-2, IL-4, TNF $\alpha$, INF $\alpha$ and NF- $\kappa B$ could impact the GR expression in many cell types ${ }^{48-50}$. Besides, the other myokines including IL-6, IL-15, TNFo, Irisin, and MSTN which could inducing thermogenesis or inhibit adipogenesis might have the ability to regulate expression of $\mathrm{GR}^{26,51-54}$. Our previous study found that myokines MSTN inhibited the differentiation of porcine adipocytes ${ }^{26}$. Hence, we detected the concentration of MSTN in muscle, and the results showed that concentration of MSTN in muscle MCM was extremely high. Next, when we treated adipocytes with MSTN, the GR $\alpha$ level was significantly reduced, in accord with the effects of MCM. MSTN also inhibited the proliferation and differentiation of porcine adipocytes, which confirmed our previous results.

DNA methylation is an important epigenetic modification mechanism and has traditionally been associated with gene repression ${ }^{55}$. Lots of studies showed that GR expression was suppressed by high levels of methylation ${ }^{27,28}$. What' more, MSTN was reported to have the ability to regulate DNA methylation levels in muscle satellite cells ${ }^{56}$. By bisulfite sequencing, we detected higher methylation levels of promoter 1-C and 1-H in IMA than SA, and these results were in accordance with the express pattern of exon 1-C and 1-H. Hence, having treated adipocytes with MSTN, we found that the main de novo methyltransferases DNMT3 $\alpha^{57}$ was induced, and the methylation levels of promoter $1-\mathrm{C}$ and $1-\mathrm{H}$ were increased.

MSTN is predominantly expressed in muscle, and was usually considered as a negative regulator of muscle growth $^{58}$. Recently, many reports found that MSTN also blocked the differentiation of adipocytes ${ }^{59,60}$. As a member of TGF- $\beta$ superfamily, MSTN works by binding to activine type II receptor. Then the Smad family members are subsequently activated and form a complex, which are then translocated to the nucleus, regulating target gene transcription ${ }^{61}$. The cooperation between Smads and AP $1^{62}, \mathrm{KLF}^{63}$ has been reported in previous studies, and the binding sites of these two important transcription factors were found in the promoter of DNMT $3 \alpha$. Thus MSTN probably regulate DNMT3a through the cooperation between Smads and these transcription factors.

In summary, we found that muscle secreted MSTN regulated the methylation levels of promoter 1-C and $1-\mathrm{H}$ in adipocytes, leading to the reduction of GR $\alpha$ level. This effect lowered the GCs sensitivity of IMA, and decreased the GCs-induced proliferation, differentiation and anti-apoptosis of adipocytes, therefore reducing fat deposition in IMA. This study provides a novel molecular mechanism of how the local myocytes inhibits adipocytes, and could contribute to specifically regulate IMA without influencing the other fat tissues deposition.

\section{Methods}

Ethics statement. All experiments were performed in accordance with the guidelines of the regional Animal Ethics Committee and were approved by the Institutional Animal Care and Use Committee of Nanjing Agricultural University (NJAU-CAST-2014-179).

Cell isolation and culture. All the animals used in this study were treated in accordance with "The Regulations on the Care for Laboratory Animals" of the Ministry of Science and Technology of the People's Republic of China. Three-day-old female Erhualian pigs were killed via intraperitoneal injection of pentobarbital sodium ( $50 \mathrm{mg} / \mathrm{kg}$ body weight) followed by exsanguinations. Backfat and longissimus dorsi (LD) muscle were isolated aseptically. The porcine pre-adipocytes were obtained through the "ceiling culture" method, isolated according to our previous report ${ }^{21}$. The backfat and LD muscle were washed and minced into approximately $1 \mathrm{~mm}^{3}$ slices, then digested with $0.1 \%(\mathrm{w} / \mathrm{v})$ Type I collagenase (Life Technologies, USA). The tubes were 
incubated for $1 \mathrm{~h}$ at $37^{\circ} \mathrm{C}$ with gentle shaking. After digestion, the cell suspension was filtrated through a 300-mm screen cloth to remove undigested tissue debris and then centrifuged at $190 \mathrm{~g}$ for $10 \mathrm{~min}$. The supernatant containing mature adipocytes was aspirated to new centrifuge tubes with DMEM and then centrifuged at $190 \mathrm{~g}$ for $10 \mathrm{~min}$. The steps above were repeated twice to obtain pure mature adipocytes in the top layer of the tube. The fatty layer was transferred to a $25 \mathrm{~cm}^{2}$ cell culture flask, which was fully filled with DMEM $+10 \%$ fetal bovine serum (FBS), inverted the flask in order to allow the floating mature adipocytes to adhere to the top inner surface, and incubated in a $37^{\circ} \mathrm{C}$ incubator with $5 \% \mathrm{CO}_{2}$ and $95 \% \mathrm{O}_{2}$. After a 12 -h incubation period, the cell media were transferred to new flasks, which were again entirely filled with DMEM $+10 \%$ FBS. This purification step was repeated twice in order to remove contaminant fibroblasts and obtain pure mature adipocytes. 10 days later, the mature adipocytes lost lipids and dedifferentiated into pre-adipocytes. Then excess cell culture media was removed and the culture flask was turned right side up. The medium was changed every 3 days until confluence was achieved.

Adipocytes differentiation. After pre-adipocytes reaching confluence, cells were induced to differentiate with the differentiation medium (DMEM, 10\% FBS, $0.25 \mathrm{nM}$ dexamethasone, $0.1 \mathrm{mM}$ 3 -isobutyl-1-methylxanthine, and $5 \mu \mathrm{g} / \mathrm{mL}$ insulin) for 3 days, then the medium was replaced with culture medium for another 9 days. All medium was refreshed every 3 days.

ACTH experiment. Twelve Xinhuai sows were randomly divided into two groups. $1 \mathrm{U} / \mathrm{kg}$ adrenocorticotropic hormone (Sigma, USA) or equivalent volume saline was injected via cervical vein per day for 9 days. Then the levels of serum cortisol were detected, and backfat and LD muscle samples were harvested. Isolated the IMAT with Ophthalmic forceps in the LD muscle.

Muscle conditioned medium. Muscle tissue was isolated from the longissimus dorsi of newborn Erhualian pigs. Ten grams of muscle tissue was minced into $1 \mathrm{~mm}^{3}$ pieces in Dulbecco's Modified Eagle's (DMEM) medium, transferred to a $25 \mathrm{~cm}^{2}$ cell culture flask, and incubated in DMEM containing $10 \%$ fetal bovine serum (FBS) at $37^{\circ} \mathrm{C}$ in a $5 \% \mathrm{CO}_{2}$ humidified atmosphere for $12 \mathrm{~h}$. The culture medium was aspirated and filtered through a $300 \mathrm{~mm}$ screen cloth to remove the tissue debris. Muscle conditioned medium was prepared by mixing culture medium in DMEM containing 10\% FBS at a ratio of 1:2.

Cell proliferation assay. Cell counting. Cell number was counted by the automated cell counter (Invitrogen, USA). Cell viability was determined using the Cell Counting Kit-8 (CCK-8) (Vazyme, Jiangsu, China) assay.

Cell viability assay. CCK-8 contains WST- 8 which can be deoxidized to the hydrosoluble formazan dye by mitochondrial dehydrogenase in living cells. Cells were treated with $10 \mu \mathrm{l} \mathrm{CCK}-8$ solutions and incubated at $37^{\circ} \mathrm{C}$ for 4 hours. The absorbance was measured using an automated microplate reader (Bio-Rad, Japan) at $450 \mathrm{~nm}$. Results were expressed as percentages of the controls, which were arbitrarily assigned with $100 \%$ viability.

EdU assay. Adipocytes were cultured with fresh growth medium containing EdU (final concentration, $10 \mathrm{mM}$ ) for 2 hours. EdU staining was conducted using Cell-Light ${ }^{\mathrm{TM}}$ EdU Apollo ${ }^{\circledR} 488$. In Vitro Imaging Kit (RiboBio, Guangzhou, China) according to the manufacturer's protocol. The EdU-labeled cells were imaged with confocal microscope.

Cell differentiation assay. Oil red O staining. Mature adipocytes were washed 3 times with PBS, and then fixed with $10 \%$ formalin for $15 \mathrm{~min}$. After fixation, the cells were washed with PBS 3 times and stained with oil red $\mathrm{O}$ for $20 \mathrm{~min}$. Subsequently, the cells were washed with $60 \%$ isopropanol for $20 \mathrm{~s}$, and then imaged with inverted microscope.

Triglyceride assay. The intra-cellular triglyceride was assayed using a triglyceride assay kit (Applygen Technologies Inc) according to the manufacture's instruction.

RT-qPCR. Total RNA was isolated from cells or tissues using TRIzol reagent (Invitrogen, Shanghai, China). cDNA for was synthesized using ProtoScript M-MuLV (NEB,USA). The DNA standards were performed using pspt 18 vector (Promega, USA) as previously described ${ }^{40}$, and primer sequences were listed in Supplementary Table 1. Real-time PCR was performed using SYBR Premix Ex Taq ${ }^{\mathrm{TM}}$ (TaKaRa, Dalian, China) on ABI StepOne Plus ${ }^{\mathrm{TM}}$ Real-Time PCR System (Applied Biosystems, USA). Relative expression was analyzed by using $2^{-\Delta \Delta \mathrm{Ct}}$ and are referred to the control treatment using RPLPO as a reference gene. The primer sequences were listed in Supplementary Table 2 .

Western blot. Total protein extracts with RIPA lysis buffer (Beyotime Biotechnology, Jiangsu, China) and protein quantities were measured by the BCA Protein Assay kit (Beyotime Biotechnology, Jiangsu, China). Antibodies against GR $\alpha$ (sc-393232, Santa Cruz, USA) and tubulin (sc-5274, Santa Cruz, USA) was used in Western blot analysis. Images were captured with VersaDoc 4000MP system (Bio-Rad).

Apoptosis and Cell Cycle Assay. After adipocytes were harvested and washed, apoptosis of adipocytes was detected with Annexin V-FITC/PI Apoptosis Detection Kit (Vazyme, Jiangsu, China) according to the manufacturer's instructions. Cell cycle were detected with Cell Cycle Assay Kit (Vazyme, Jiangsu, China). The Cells were 
sorted by fluorescence-activated cell sorting using a Beckman Coulter instrument (Becton Dickinson, Franklin, NJ, USA). The early apoptosis rate was calculated as AnnexinV (+) PI (-) cells/total cell $\times 100 \%$, and the late apoptosis rate was calculated as Annexin V $(+) \mathrm{PI}(+)$ cells/total cell $\times 100 \%$, and the necrosis rate was calculated as Annexin V ( - ) PI $(+)$ cells/total cell $\times 100 \%$.

ELISA. The commercial ELISA kits (R\&D Systems, USA) was used to measure the cortisol concentration in porcine serum according to the manufacturer's instruction. The porcine MSTN ELISA Kit (Elabscience, Wuhan, China) was used to determine the concentration of MSTN in MCM and serum according to the manufacturer's instruction.

Methylation analysis. Bisulfite sequencing. Genomic DNA was extracted using the phenol-chloroform method from SA and IMA pre-adipocytes. Bisulfite treatment was performed according to the manufacturer's instruction of EZ DNA Methylation-Gold Kit ${ }^{\mathrm{TM}}$ (Zymo Research, USA). Primers (BSP- Promoter 1 C, BSPPromoter $1 \mathrm{H}$ ) for the amplification of the GR promoter region were designed by Methyl Primer Express v1.0. The PCR products were cloned in the pEASY-T3 Cloning Vector (Transgen Biotech, Beijing, China), then positive clones were sequenced.

Methylated DNA immunoprecipitation. This method was carried out followed the previously description $^{64}$. Briefly speaking, $40 \mu \mathrm{g}$ of DNA was sheared between $200 \sim 500$ bp by sonication, then immunoprecipitated by $5 \mu \mathrm{l} 5$-mC monoclonal antibody (Diagenode, Belgian). Ct values were determined by RT-qPCR, and the Methylation Ratio was calculated by the formula Methylation Ratio (IP/Input) $=2^{-(\mathrm{Ct}[\mathrm{IP}]-\mathrm{Ct}[\mathrm{Input}])} \times 100 \%$.

Statistical analysis. At least three biological replicates were used for each analysis. Statistically significant differences were determined using paired t-test or one-way ANOVA of variance using SPSS 18.0 software (SPSS Inc., USA). P-value of 0.05 was considered significant. All values are presented as the mean $\pm \mathrm{SEM}$.

\section{References}

1. Tran, T. T. \& Kahn, C. R. Transplantation of adipose tissue and stem cells: role in metabolism and disease. Nat Rev Endocrinol 6, 195-213 (2010)

2. Hovenier, R., Kanis, E., Asseldonk, T. V. \& Westerink, N. G. Genetic parameters of pig meat quality traits in a halothane negative population. Livestock Production Science 32, 309-321 (1992).

3. Kouba, M. \& Sellier, P. A review of the factors influencing the development of intermuscular adipose tissue in the growing pig. Meat science 88, 213-220 (2011).

4. Kouba, M. \& Bonneau, M. Compared development of intermuscular and subcutaneous fat in carcass and primal cuts of growing pigs from 30 to $140 \mathrm{~kg}$ body weight. Meat science 81, 270-274 (2009).

5. Wang, S. et al. Glucose utilization, lipid metabolism and BMP-Smad signaling pathway of porcine intramuscular preadipocytes compared with subcutaneous preadipocytes. Cellular physiology and biochemistry: international journal of experimental cellular physiology, biochemistry, and pharmacology 31, 981-996 (2013).

6. Maela, K., Laurent, L. B., Jacques, M., Jean, N. \& Jaap, V. M. Deposition of dietary fatty acids and of de novo synthesised fatty acids in growing pigs: effects of high ambient temperature and feeding restriction. British Journal of Nutrition 93, 803-811 (2005).

7. Gardan, D., Gondret, F. \& Louveau, I. Lipid metabolism and secretory function of porcine intramuscular adipocytes compared with subcutaneous and perirenal adipocytes. American journal of physiology. Endocrinology and metabolism 291, E372-E380 (2006).

8. Zhu, Y. et al. Inflammation and the depot-specific secretome of human preadipocytes. Obesity 23, 989-999 (2015).

9. Zhou, G. et al. Global comparison of gene expression profiles between intramuscular and subcutaneous adipocytes of neonatal landrace pig using microarray. Meat science 86, 440-450 (2010).

10. Lundgren, M., Buren, J., Ruge, T., Myrnas, T. \& Eriksson, J. W. Glucocorticoids down-regulate glucose uptake capacity and insulinsignaling proteins in omental but not subcutaneous human adipocytes. J Clin Endocr Metab 89, 2989-2997 (2004).

11. Louveau, I. \& Gondret, F. GH and insulin affect fatty acid synthase activity in isolated porcine adipocytes in culture without any modifications of sterol regulatory element binding protein-1 expression. The Journal of endocrinology 181, 271-280 (2004).

12. Barclay, J. L. et al. Effects of glucocorticoids on human brown adipocytes. The Journal of endocrinology 224, 139-147 (2015).

13. Bader, T. et al. Human adipose tissue under in vitro inhibition of 11beta-hydroxysteroid dehydrogenase type 1: differentiation and metabolism changes. Horm Metab Res 34, 752-757 (2002).

14. Floyd, Z. E. \& Stephens, J. M. STAT5A promotes adipogenesis in nonprecursor cells and associates with the glucocorticoid receptor during adipocyte differentiation. Diabetes 52, 308-314 (2003).

15. Wu, Z., Bucher, N. \& Farmer, S. R. Induction of peroxisome proliferator-activated receptor gamma during the conversion of 3 T3 fibroblasts into adipocytes is mediated by C/EBPbeta, C/EBPdelta, and glucocorticoids. Molecular and cellular biology 16, 4128-4136 (1996).

16. Veilleux, A. et al. Expression of genes related to glucocorticoid action in human subcutaneous and omental adipose tissue. The Journal of steroid biochemistry and molecular biology 122, 28-34 (2010).

17. Wajchenberg, B. L. et al. Estimation of body fat and lean tissue distribution by dual energy X-ray absorptiometry and abdominal body fat evaluation by computed tomography in Cushing's disease. J Clin Endocr Metab 80, 2791-2794 (1995).

18. Rosmond, R., Holm, G. \& Björntorp, P. Food-induced cortisol secretion in relation to anthropometric, metabolic and haemodynamic variables in men. International Journal of Obesity \& Related Metabolic Disorders Journal of the International Association for the Study of Obesity 24, 416-422 (2000).

19. Rosmond, R., Dallman, M. F. \& BjRntorp, P. Stress-related cortisol secretion in men: relationships with abdominal obesity and endocrine, metabolic and hemodynamic abnormalities. J Clin Endocr Metab 83, 1853-1859 (1998).

20. Geer, E. B. et al. MRI assessment of lean and adipose tissue distribution in female patients with Cushing's disease. Clinical endocrinology 73, 469-475 (2010).

21. Sun, W. et al. Acquisition of pig intramuscular preadipocytes through dedifferentiation of mature adipocytes and establishment of optimal induction conditions. Genetics and molecular research: GMR 12, 5926-5936 (2013).

22. Lee, M. J., Gong, D. W., Burkey, B. F. \& Fried, S. K. Pathways regulated by glucocorticoids in omental and subcutaneous human adipose tissues: a microarray study. American journal of physiology. Endocrinology and metabolism 300, E571-E580 (2011).

23. Rosen, E. D., Walkey, C. J., Puigserver, P. \& Spiegelman, B. M. Transcriptional regulation of adipogenesis. Genes \& development 14, 1293-1307 (2000).

24. Slavin, B. G., Ong, J. M. \& Kern, P. A. Hormonal regulation of hormone-sensitive lipase activity and mRNA levels in isolated rat adipocytes. Journal of lipid research 35, 1535-1541 (1994). 
25. Villena, J. A., Roy, S., Sarkadi-Nagy, E., Kim, K. H. \& Sul, H. S. Desnutrin, an adipocyte gene encoding a novel patatin domaincontaining protein, is induced by fasting and glucocorticoids: ectopic expression of desnutrin increases triglyceride hydrolysis. The Journal of biological chemistry 279, 47066-47075 (2004).

26. Sun, W. et al. Myostatin inhibits porcine intramuscular preadipocyte differentiation in vitro. Domestic animal endocrinology 55, 25-31 (2016).

27. Perroud, N. et al. Increased methylation of glucocorticoid receptor gene (NR3C1) in adults with a history of childhood maltreatment: a link with the severity and type of trauma. Translational psychiatry $\mathbf{1}$, e59 (2011).

28. Hompes, T. et al. Investigating the influence of maternal cortisol and emotional state during pregnancy on the DNA methylation status of the glucocorticoid receptor gene (NR3C1) promoter region in cord blood. Journal of psychiatric research 47, 880-891 (2013).

29. Satoor, S. N. et al. Location, location, location: Beneficial effects of autologous fat transplantation. Scientific reports 1, 81 (2011)

30. Kovalik, J. P. et al. Metabolic remodeling of human skeletal myocytes by cocultured adipocytes depends on the lipolytic state of the system. Diabetes 60, 1882-1893 (2011).

31. Gondret, F., Guitton, N., Guillermregost, C. \& Louveau, I. Regional differences in porcine adipocytes isolated from skeletal muscle and adipose tissues as identified by a proteomic approach. Journal of animal science 86, 2115-2125 (2008).

32. Zhang, G. H. et al. Comparison of the adipogenesis in intramuscular and subcutaneous adipocytes from Bamei and Landrace pigs. Biochemistry and cell biology 92, 259-267 (2014).

33. Iida, S. et al. Primary Cortisol Resistance Accompanied by a Reduction in Glucocorticoid Receptors in 2 Members of the Same Family. J Clin Endocr Metab 60, 967-971 (1985).

34. Ansari, N. N. et al. Vitamin D decreases in vitro glucocorticoid sensitivity via down regulation of glucocorticoid receptor expression. Endocrine Abstracts 37, OC5.2 (2015).

35. Sun, X.-J. et al. Combination of erythromycin and dexamethasone improves corticosteroid sensitivity induced by CSE through inhibiting PI3K- $\delta /$ Akt pathway and increasing GR protein. American Journal of Physiology-Lung Cellular and Molecular Physiology 309(2), L139-L146 (2015).

36. Shahidi, H. et al. Imbalanced expression of the glucocorticoid receptor isoforms in cultured lymphocytes from a patient with systemic glucocorticoid resistance and chronic lymphocytic leukemia. Biochemical and biophysical research communications 254, 559-565 (1999).

37. Russcher, H. et al. Associations between promoter usage and alternative splicing of the glucocorticoid receptor gene. Journal of molecular endocrinology 38, 91-98 (2007).

38. Zou, H. et al. Breed-dependent transcriptional regulation of 5'-untranslated GR (NR3C1) exon $1 \mathrm{mRNA}$ variants in the liver of newborn piglets. PloS one 7, e40432 (2012).

39. Sinclair, D., Webster, M. J., Fullerton, J. M. \& Weickert, C. S. Glucocorticoid receptor mRNA and protein isoform alterations in the orbitofrontal cortex in schizophrenia and bipolar disorder. BMC psychiatry 12, 84 (2012).

40. Jiang, Z. et al. Porcine glucocorticoid receptor (NR3C1) gene: tissue-specificity of transcriptional strength and glucocorticoid responsiveness of alternative promoters. The Journal of steroid biochemistry and molecular biology 141, 87-93 (2014).

41. Reyer, H., Ponsuksili, S., Wimmers, K. \& Murani, E. Transcript variants of the porcine glucocorticoid receptor gene (NR3C1). General and comparative endocrinology 189, 127-133 (2013).

42. Turner, J. D., Vernocchi, S., Schmitz, S. \& Muller, C. P. Role of the $5^{\prime}$-untranslated regions in post-transcriptional regulation of the human glucocorticoid receptor. Bba-Gene Regul Mech 1839, 1051-1061 (2014).

43. Yan, J., Gan, L., Yang, H. L. \& Sun, C. The proliferation and differentiation characteristics of co-cultured porcine preadipocytes and muscle satellite cells in vitro. Mol Biol Rep 40, 3197-3202 (2013).

44. Dietze, D. et al. Impairment of insulin signaling in human skeletal muscle cells by co-culture with human adipocytes. Diabetes 51, 2369-2376 (2002).

45. Uezumi, A., Fukada, S., Yamamoto, N., Takeda, S. \& Tsuchida, K. Mesenchymal progenitors distinct from satellite cells contribute to ectopic fat cell formation in skeletal muscle. Nature cell biology 12, 143-152 (2010).

46. Chu, W. et al. $\mathrm{C} 2 \mathrm{C} 12$ myotubes inhibit the proliferation and differentiation of 3T3-L1 preadipocytes by reducing the expression of glucocorticoid receptor gene. Biochemical and biophysical research communications 472, 68-74 (2016).

47. Welc, S. S. \& Clanton, T. L. The regulation of interleukin-6 implicates skeletal muscle as an integrative stress sensor and endocrine organ. Experimental Physiology 98, 359-371 (2013).

48. Pace, T. W. W., Fang, H. \& Miller, A. H. Cytokine-effects on glucocorticoid receptor function: Relevance to glucocorticoid resistance and the pathophysiology and treatment of major depression. Brain Behavior \& Immunity 21, 9-19 (2007).

49. Pariante, C. M. et al. The Proinflammatory Cytokine, Interleukin-1 $\alpha$, Reduces Glucocorticoid Receptor Translocation and Function 1. Endocrinology 140, 4359-4366 (1999).

50. Mckay, L. I. Molecular Control of Immune/Inflammatory Responses: Interactions Between Nuclear Factor- B and Steroid ReceptorSignaling Pathways. Endocrine Reviews 20, 435-459 (1999).

51. Lightfoot, A. P. et al. Skeletal Muscle as an Endocrine organ; the effect of TNF-alpha. Faseb Journal 24, 816-3 (2010).

52. Pedersen, B. K. \& Febbraio, M. A. Muscle as an endocrine organ: focus on muscle-derived interleukin-6. Physiological reviews $\mathbf{8 8}$, 1379-1406 (2008).

53. Quinn, L. S., Anderson, B. G., Strait-Bodey, L., Stroud, A. M. \& Argiles, J. M. Oversecretion of interleukin-15 from skeletal muscle reduces adiposity. American journal of physiology. Endocrinology and metabolism 296, E191-E202 (2009).

54. Zhang, F. et al. PPAR $\gamma$ and MyoD are differentially regulated by myostatin in adipose-derived stem cells and muscle satellite cells. Biochemical and biophysical research communications 458, 375-380 (2015).

55. Dahlman, I. et al. The fat cell epigenetic signature in post-obese women is characterized by global hypomethylation and differential DNA methylation of adipogenesis genes. Int J Obes (Lond) 39, 910-919 (2015).

56. Zhang, F. et al. PPARgamma and MyoD are differentially regulated by myostatin in adipose-derived stem cells and muscle satellite cells. Biochemical and biophysical research communications 458, 375-380 (2015).

57. Kato, Y. et al. Role of the Dnmt3 family in de novo methylation of imprinted and repetitive sequences during male germ cell development in the mouse. Human molecular genetics 16, 2272-2280 (2007).

58. McPherron, A. C., Lawler, A. M. \& Lee, S. J. Regulation of skeletal muscle mass in mice by a new TGF-beta superfamily member. Nature 387, 83-90 (1997)

59. Dominique, J. E. \& Gerard, C. Myostatin regulation of muscle development: Molecular basis, natural mutations, physiopathological aspects. Exp Cell Res 312, 2401-2414 (2006).

60. Rebbapragada, A., Benchabane, H., Wrana, J. L., Celeste, A. J. \& Attisano, L. Myostatin signals through a transforming growth factor beta-like signaling pathway to block adipogenesis. Mol Cell Biol 23, 7230-7242 (2003).

61. Joulia-Ekaza, D. \& Cabello, G. The myostatin gene: physiology and pharmacological relevance. Curr Opin Pharmacol 7, 310-315 (2007).

62. Omata, Y. et al. Genomewide Comprehensive Analysis Reveals Critical Cooperation Between Smad and c-Fos in RANKL-Induced Osteoclastogenesis. J Bone Miner Res 30, 869-877 (2015).

63. Guo, P., Xing, C. S., Dong, X. Y. \& Dong, J. T. Ras signaling interrupts TGF-beta-induced KLF5 acetylation to alter the KLF5-Smads transcriptional assembly in epithelial cells. Cancer Res 72, Lb-23 (2012)

64. Willems, E. et al. Differential Expression of Genes and DNA Methylation associated with Prenatal Protein Undernutrition by Albumen Removal in an avian model. Scientific reports 6 (2016). 


\section{Acknowledgements}

This study was supported by National Natural Science Foundation of China (No. 31272423), and National Key Technology Support Program (No. 2015BAD03B01).

\section{Author Contributions}

J.C., W.W.C., W.W., and L.F.Z. conceived and designed the project. W.W.C., W.W., H.Y.H., Y.G., K.Q.L., Y.T., and Z.H.J performed all the experiments and prepared the figures. W.W.C. and W.W. wrote the manuscript. All authors reviewed the manuscript.

\section{Additional Information}

Supplementary information accompanies this paper at doi:10.1038/s41598-017-00615-9

Competing Interests: The authors declare that they have no competing interests.

Publisher's note: Springer Nature remains neutral with regard to jurisdictional claims in published maps and institutional affiliations.

(c) (i) This work is licensed under a Creative Commons Attribution 4.0 International License. The images or other third party material in this article are included in the article's Creative Commons license, unless indicated otherwise in the credit line; if the material is not included under the Creative Commons license, users will need to obtain permission from the license holder to reproduce the material. To view a copy of this license, visit http://creativecommons.org/licenses/by/4.0/

(c) The Author(s) 2017 